\title{
On the Blow-up of Solutions of the 3-D Euler Equations in a Bounded Domain
}

\author{
Andrew B. Ferrari \\ Department of Mathematics, Duke University, Durham, NC 27706, USA
}

Received July 7, 1992; in revised form October 6, 1992

\begin{abstract}
It is shown that if $[0, \hat{T})$ is the maximal interval of existence of a smooth solution $u$ of the incompressible Euler equations in a bounded, simply connected domain $\Omega \subseteq \mathbf{R}^{3}$, then $\int_{0}^{\hat{T}}|\omega(\cdot, t)|_{L^{\infty}(\Omega)} d t=\infty$, where $\omega=\nabla \times u$ is the vorticity. Crucial to this result is a special estimate proven in $\Omega$ of the maximum velocity gradient in terms of the maximum vorticity and a logarithmic term involving a higher norm of the vorticity.
\end{abstract}

\section{Introduction}

The Euler equations are a system of nonlinear partial differential equations that describe inviscid, incompressible fluid flow. For flow in a bounded domain $\Omega \subseteq \mathbf{R}^{3}$, the appropriate initial-boundary value problem has a unique smooth solution for a short time, provided the initial data is sufficiently smooth. It is not known whether this smooth solution persists for all time or if it becomes singular at some later time, but the long-standing conjecture is that it indeed becomes singular due to the development of turbulence in the flow. In this paper it is proved that if $[0, \hat{T})$ is the maximal interval of existence of such a solution $u$, specifically of the class $C\left([0, T] ; H^{s}(\Omega)\right)$, with $s \geqq 3$ and $T<\hat{T}$, then

$$
\int_{0}^{\hat{T}}|\omega(\cdot, t)|_{L^{\infty}(\Omega)} d t=\infty
$$

and in particular $\sup _{t \in[0, \hat{T}]}|\omega(\cdot, t)|_{L^{\infty}(\Omega)}=\infty$. Here $\omega=\nabla \times u$ is the vorticity of the flow. The significance of this result is that it isolates one specific singularity responsible for the loss of smoothness of the velocity.

Crucial to this result is the special estimate

$$
|u|_{W^{1, \infty}(\Omega)} \leqq C\left(1+\log +\frac{|\omega|_{H^{2}(\Omega)}}{|\omega|_{L^{\infty}(\Omega)}}\right)|\omega|_{L^{\infty}(\Omega)} .
$$


This estimate appears to be new in case $\Omega$ is a bounded domain. In [7] Kato and Ponce proved it for $\Omega=\mathbf{R}^{n}$ and $u \in W^{s, p}\left(\mathbf{R}^{n}\right)$, and earlier, in [4], Beale, Kato, and Majda had obtained a slightly less refined form of it for $\Omega=\mathbf{R}^{3}$ and $u \in H^{s}\left(\mathbf{R}^{3}\right)$. More recently, M. Taylor in [13] derived a generalization of it along with several variants, for $\Omega=\mathbf{R}^{n}$, within the context of pseudodifferential operators. For $\Omega \in \mathbf{R}^{3}$ a bounded domain, there does appear in [18] a weaker version of the estimate in which the logarithmic term is raised to the seventh power, but it is not sufficient to obtain the result of this paper. It is worth pointing out that in [15] and [16], $L^{p}$ estimates, for finite $p$, of the velocity gradient in terms of the divergence and curl of the velocity, are shown to hold under certain topological conditions on the domain $\Omega$.

The result of this paper, but for flow in all of $\mathbf{R}^{3}$, was obtained in 1984 by Beale, Kato, and Majda [4], utilizing such techniques as the Fourier transform and the Biot-Savart law. The difficulty in proving this result for a bounded domain is that entirely different techniques must be utilized. One result that has been obtained in this latter case is by M. Zajaczkowski [18], who in 1988 showed that a smooth solution of the Euler equations in a bounded domain may be continued if the time integral of the maximum vorticity does not exceed a specific quantity determined by a high norm of the initial data. This result allows for the possibility of singularities other than $|\omega|_{\infty}$ being responsible for the loss of smoothness of the velocity. Recently, T. Yanagisawa [17] independently obtained the result of this paper for bounded domains in $\mathbf{R}^{n}$ with smooth boundary and general topology, including a corresponding version of the special estimate described above, utilizing a different method. An advantage of the method is that it yields the special estimate and hence the result in a more general context, and an advantage of the method of this paper is that it can be adapted to yield the analogous estimate for solutions of more general elliptic boundary value problems.

The following definitions, notation, and results will be used throughout the paper. For $s \in \mathbf{Z}^{+}$and $1 \leqq p \leqq \infty$, we define the usual Sobolev spaces $W^{s, p}(\Omega)=$ $\left\{u \in L^{p}(\Omega): D^{\beta} u \in L^{p}(\Omega),|\bar{\beta}| \leqq s\right\}$, the derivatives being in the distributional sense. These spaces are equipped with the norms

$$
\begin{aligned}
|u|_{W^{s, p}(\Omega)} & =\left(\sum_{|\beta| \leqq s}\left|D^{\beta} u\right|_{L^{p}(\Omega)}^{p}\right)^{1 / p}, \quad 1 \leqq p<\infty, \\
|u|_{W^{s, \infty}(\Omega)} & =\sum_{|\beta| \leqq s}\left|D^{\beta} u\right|_{L^{\infty}(\Omega)} .
\end{aligned}
$$

When $p=2$, the corresponding spaces will be denoted $H^{s}(\Omega)$. We shall also define the Sobolev spaces $W^{s-1 / p, p}(\partial \Omega)$, for $s \in \mathbf{Z}^{+}$and $1 \leqq p<\infty$, to consist of all functions $f$ defined on $\partial \Omega$ which are restrictions to $\partial \Omega$ of functions $F \in W^{s, p}(\Omega)$. These spaces are normed by $|f|_{W^{s-1 / p, p}(\partial \Omega)}=\inf |F|_{W^{s, p}(\Omega)}$, the infimum being taken over all such $F$. There will be no notational distinction between Sobolev spaces of scalar- or vector-valued functions. The Sobolev Embedding Theorem will be necessary in several instances, and for these it suffices to note from the theorem that if $\Omega \subseteq \mathbf{R}^{n}$ has $C^{1}$ boundary, then $W^{s, p}(\Omega) \subseteq L^{p}(\Omega)$ continuously if $s<n / p$ and $p \leqq q \leqq n p /(n-s p)$, and $W^{s, p}(\Omega) \subseteq C^{k}(\bar{\Omega})$ continuously if $s>k+n / p$. Two special cases will be used rather frequently; namely, for $\Omega \subseteq \mathbf{R}^{3}, H^{1}(\Omega) \subseteq L^{6}(\Omega)$ and $W^{1, p}(\Omega) \subseteq L^{\infty}(\Omega)$, if $p>3$. 
The plan of the paper is as follows. In Sect. 1 the main result is stated and proved, except for the proof of the special estimate. In Sect. 2 the special estimate is stated and proved in a slightly more general form.

The work of this paper was completed as part of the author's Ph.D. thesis [5], under the direction of $\mathrm{J}$. Thomas Beale.

\section{Main Result}

Necessary to this section is the following short-time existence result for solutions of the Euler equations (1)-(3) below, in a bounded domain $\Omega \subseteq \mathbf{R}^{3}$, which has been adapted from Theorem 1 of [6] (see also [14]).

$$
\begin{aligned}
u_{t}+(u \cdot \nabla) u+\nabla p=0 & \text { in } \Omega, \\
\nabla \cdot u=0 & \text { in } \Omega, \\
u \cdot n=0 & \text { on } \partial \Omega .
\end{aligned}
$$

Theorem 1. If $\Omega \subseteq \mathbf{R}^{3}$ is a bounded domain with smooth boundary $\partial \Omega$, and if $u_{0} \in H^{s}(\Omega), s \geqq 3$, with $\nabla \cdot u_{0}=0$ and $\left.u_{0} \cdot n\right|_{\partial \Omega}=0$, then there exist a time $T^{*}>0$ and a constant $C$, both depending only on $\left|u_{0}\right|_{H^{3}(\Omega)}$, and unique functions $u \in C\left(\left[0, T^{*}\right] ; H^{s}(\Omega)\right)$ and $p \in C\left(\left[0, T^{*}\right] ; H^{s+1}(\Omega)\right)$ satisfying $(1)-(3)$ for $t \in\left[0, T^{*}\right]$, such that $\sup _{t \in\left[0, T^{*}\right]}|u(\cdot, t)|_{s} \leqq C\left|u_{0}\right|_{s}$.

We now state the main result of the paper.

Theorem 2. If $u$ is a solution of the Euler equations (1)-(3) in the class $C\left([0, T] ; H^{s}(\Omega)\right), s \geqq 3$, with $\Omega \subseteq \mathbf{R}^{3}$ a bounded, simply connected domain with smooth boundary, and if $T=\hat{T}$ is the first time such that $u$ is not contained in this class, then

$$
\int_{0}^{\hat{T}}|\omega(\cdot, t)|_{L^{\infty}(\Omega)} d t=\infty ;
$$

and, in particular, $\sup _{t \in(0, \hat{T})}|\omega(\cdot, t)|_{L^{\infty}(\Omega)}=\infty$, where $\omega=\nabla \times u$ is the vorticity.

Proof. The proof is by contradiction. In short, we assume that

$$
\int_{0}^{\hat{T}}|\omega(\cdot, t)|_{L^{\infty}(\Omega)} d t=M<\infty,
$$

and show that this implies that $u(x, \hat{T})$ is contained in $H^{s}(\Omega)$, contradicting the hypothesis of the theorem.

The derivation of the contradiction consists of four steps, essentially. First we derive an energy estimate for smooth $u$ of the form

$$
\log |u(\cdot, t)|_{s} \leqq \log \left|u_{0}\right|_{s}+C \int_{0}^{T}|u(\cdot, t)|_{1, \infty} d t,
$$

and then we derive a special estimate of elliptic type of the form

$$
|u(\cdot, t)|_{W^{1, \infty}(\Omega)} \leqq C\left[\left(1+\log ^{+}|u(\cdot, t)|_{H^{s}(\Omega)}\right)|\omega(\cdot, t)|_{L^{\infty}(\Omega)}+1\right] .
$$


We then apply Gronwall's inequality to a combination of these two estimates to obtain

$$
|u(\cdot, t)|_{H^{s}(\Omega)} \leqq K, t \in[0, \hat{T}],
$$

for smooth $u$, and we use this estimate together with the short-time existence theorem and an approximation procedure to conclude that $u(x, \hat{T}) \in H^{s}(\Omega)$ for $u$ a priori in the proper class.

We proceed with the derivation of (5). Assuming for now that $u \in$ $C\left([0, T] ; H^{s+1}(\Omega)\right), T \leqq \hat{T}$, we apply $D^{\alpha},|\alpha| \leqq s$, to both sides of (1), multiply by $D^{\alpha} u$, and integrate over $\Omega$ to obtain

$$
\begin{gathered}
\frac{1}{2} \frac{d}{d t}\left|D^{\alpha} u(\cdot, t)\right|_{L^{2}}+\left(u \cdot D^{\alpha} \nabla u, D^{\alpha} u\right)_{L^{2}}+\left(D^{\alpha} u, D^{\alpha} \nabla p\right)_{L^{2}} \\
=\left(u \cdot D^{\alpha} \nabla u-D^{\alpha}(u \cdot \nabla u), D^{\alpha} u\right)_{L^{2}},
\end{gathered}
$$

and since $\nabla \cdot u=0$ in $\Omega$ and $u \cdot n=0$ on $\partial \Omega$, the second term vanishes, and we have

$$
\frac{1}{2} \frac{d}{d t}\left|D^{\alpha} u(\cdot, t)\right|_{L^{2}}^{2} \leqq\left|D^{\alpha} u\right|_{L^{2}}\left[\left|u \cdot D^{\alpha} \nabla u-D^{\alpha}(u \cdot \nabla u)\right|_{L^{2}}+\left|D^{\alpha} \nabla p\right|_{L^{2}}\right] .
$$

The difference term on the right-hand side is estimated with the help of a lemma.

Lemma 1. i) If $f, g \in H^{s}(\Omega) \cap C(\Omega)$, then

$$
|f g|_{H^{s}(\Omega)} \leqq C\left(|f|_{H^{s}(\Omega)}|g|_{L^{\infty}(\Omega)}+|f|_{L^{\infty}(\Omega)}|g|_{H^{s}(\Omega)}\right) .
$$

ii) If $f \in H^{s}(\Omega) \cap C^{1}(\Omega)$ and $g \in H^{s-1}(\Omega) \cap C(\Omega)$, then for $|\alpha| \leqq s$,

$$
\left|D^{\alpha}(f g)-f D^{\alpha} g\right|_{L^{2}(\Omega)} \leqq C\left(|f|_{H^{s}(\Omega)}|g|_{L^{\infty}(\Omega)}+|f|_{W^{1, \infty}(\Omega)}|g|_{H^{s-1}(\Omega)}\right) .
$$

Proof. These estimates are easily derived using extension operators, well-known to be bounded on the necessary spaces, together with the free space versions of the estimates in $[4,8$, and 9$]$.

Applying to (8) the second part of the lemma with $f=u$ and $g=\nabla u$ implies

$$
\frac{1}{2} \frac{d}{d t}\left|D^{\alpha} u(\cdot, t)\right|_{L^{2}}^{2} \leqq C\left|D^{\alpha} u\right|_{L^{2}}\left(|u|_{s}|u|_{W^{1, \infty}}+\left|D^{\alpha} \nabla p\right|_{L^{2}}\right) .
$$

And, summing over $|\alpha| \leqq s$, we obtain

$$
\frac{1}{2} \frac{d}{d t}|u(\cdot, t)|_{s}^{2} \leqq C\left(|u|_{s}^{2}|u|_{W^{1, \infty}}+|u|_{s}|\nabla p|_{s}\right) .
$$

The pressure term is estimated with the aid of another lemma.

Lemma 2. If $u \in H^{s}(\Omega)$ and $p \in H^{s+1}(\Omega), s \geqq 3$, then

$$
|\nabla p|_{s} \leqq C|u|_{s}|u|_{1, \infty} .
$$

Proof. The argument given here is a modification of an argument in [14]. There, $|\nabla p|_{s}$ is estimated in terms of $|u|_{s}^{2}$, which is not sufficient for our purposes. Proceeding with the proof, we first take the divergence of (1) and the dot product of 
the same with $n=\left(n_{1}, n_{2}, n_{3}\right)$, and use (2) and (3) to obtain the following Neumann problem for $p$ :

$$
\begin{aligned}
& \nabla p=-\sum_{i, j} D_{j} u_{i} \cdot D_{i} u_{j} \quad \text { in } \Omega, \\
& \frac{\partial p}{\partial n}=-\sum_{i, j} u_{i}\left(D_{i} u_{j}\right) n_{j} \quad \text { on } \partial \Omega .
\end{aligned}
$$

The derivative of $u$ on the right side of (11) may be eliminated by representing $\partial \Omega$ locally as a smooth function $\phi(x)=0$, as in [14], so that on each local patch of $\partial \Omega(11)$ becomes

where $\psi_{i j}=D_{i j} \phi /|\nabla \phi|$.

$$
\frac{\partial p}{\partial n}=-\sum_{i, j} u_{i} u_{j} \psi_{i j}
$$

We estimate $\nabla p$ by applying the Trace theorem and the first part of Lemma 1 to a standard estimate of $\nabla p$, derived by the usual method of choosing a partition of unity for $\Omega$, deriving local estimates from (10) and (12), and combining them for the global estimate:

$$
\begin{aligned}
|\nabla p|_{H^{s}(\Omega)} \leqq & C\left(\left|\sum_{i, j} D_{j} u_{i} \cdot D_{i} u_{j}\right|_{H^{s-1}(\Omega)}+\left|\sum_{i, j} u_{i} u_{j} \psi_{i j}\right|_{H^{s-1 / 2}(\partial \Omega)}\right) \\
\leqq & C\left[\sum_{i, j}\left(\left|D_{j} u_{i}\right|_{s-1}\left|D_{i} u_{j}\right|_{\infty}+\left|D_{j} u_{i}\right|_{\infty}\left|D_{i} u_{j}\right|_{s-1}\right)\right. \\
& \left.+\sum_{i, j}\left(\left|u_{i}\right|_{s}\left|u_{j}\right|_{\infty}+\left|u_{i}\right|_{\infty}\left|u_{j}\right|_{s}\right)\right] \\
\leqq & C|u|_{s}|u|_{1, \infty} .
\end{aligned}
$$

Applying Lemma 2 to (9) we obtain

$$
\frac{1}{2} \frac{d}{d t}|u(t)|_{s}^{2} \leqq C|u|_{s}^{2}|u|_{1, \infty},
$$

so that by Gronwall's inequality,

$$
|u(t)|_{s} \leqq\left|u_{0}\right|_{s} \exp \left(C \int_{0}^{T}|u(t)|_{1, \infty} d t\right), t \in[0, T] .
$$

Taking the logarithm of both sides then yields (5) for $t \in[0, T]$.

The derivation of (6) will be deferred to Sect. 2, where the estimate is stated and proved as a Corollary to Proposition 1.

In order to combine (5) and (6) so that Gronwall's inequality may be applied to yield (7), we rewrite the estimates in the following way:

$$
\begin{gathered}
\log \left(|u(\cdot, t)|_{s}+e\right) \leqq \log \left(\left|u_{0}\right|_{s}+e\right)+C \int_{0}^{T}|u(\cdot, t)|_{1, \infty} d t \\
|u(\cdot, t)|_{W^{1, \infty}(\Omega)} \leqq 2 C\left[|\omega(\cdot, t)|_{L^{\infty}(\Omega)} \log \left(|u(\cdot, t)|_{H^{s}(\Omega)}+e\right)+1\right] .
\end{gathered}
$$


Combining these we have

$$
\log U(t) \leqq \log U(0)+C \int_{0}^{T}\left(1+|\omega(\cdot, t)|_{L^{\infty}(\Omega)} \log U(t)\right) d t,
$$

where $U(t)=|u(\cdot, t)|_{H^{s}(\Omega)}+e$. And now since $T \leqq \hat{T}$ an application of Gronwall's inequality yields

$$
\log U(t) \leqq F(\hat{T})+C \int_{0}^{\hat{T}}|\omega(\cdot, t)|_{L^{\infty}(\Omega)} F(t) \exp \left(C \int_{t}^{\hat{T}}|\omega(\cdot, s)|_{L^{\infty}(\Omega)} d s\right) d t,
$$

where $F(t)=U(0)+C t$. Taking exponentials, we finally obtain

$$
|u(\cdot, t)|_{H^{s}(\Omega)} \leqq K, \quad t \in[0, T]
$$

where

$$
K=U(0) e^{C \hat{T}} \exp \left\{C \int_{0}^{\hat{T}}|\omega(\cdot, t)|_{L^{\infty}(\Omega)} F(t) \exp \left(C \int_{t}^{\hat{T}}|\omega(\cdot, s)|_{L^{\infty}(\Omega)} d s\right) d t\right\} .
$$

Note that $K<\infty$ because of (4).

Estimate (17) has been derived for $u \in C\left([0, T] ; H^{s+1}(\Omega)\right)$ and $T \leqq \hat{T}$, but it actually implies that $u(\hat{T}) \in H^{s}(\Omega)$ for $u$ a priori only in $C\left([0, \hat{T}) ; H^{s}(\bar{\Omega})\right)$. To see this, start with $u_{0} \in H^{s}(\Omega)$, which from (17) is clearly bounded by $2 K$. Theorem 1 then guarantees the existence of an interval $\left[0, T_{1}\right]$ on which $u(t)$ is in $H^{s}(\Omega)$, with $T_{1}$ depending only on $2 K$. Now approximate $u_{0}$ in $H^{s}(\Omega)$ by a sequence $u_{0}^{n} \in H^{s+1}(\Omega)$ satisfying $\left.u_{0}^{n} \cdot n\right|_{\partial}=0$ and $\nabla \cdot u_{0}^{n}=0$, which is possible by the continuity in $H^{s}(\Omega)$ of the projection onto divergence-free vectors tangential to $\partial \Omega$. Theorem 1 thus gives rise to a sequence of solutions $u^{n}(t) \in H^{s+1}(\Omega)$, also for $t \in\left[0, T_{1}\right]$ for $n$ sufficiently large, since $T_{1}$ depends particularly on $\left|u_{0}^{n}\right|_{3}$, and $\left|u_{0}^{n}\right|_{3} \leqq\left|u_{0}^{n}\right|_{s} \leqq 2 K$ for $n$ sufficiently large. Thus we may derive (14) for $u^{n}$ as before, with $T=T_{1}$ :

$$
\left|u^{n}(t)\right|_{s} \leqq\left|u_{0}^{n}\right|_{s} \exp \left(C \int_{0}^{T_{1}}\left|u^{n}(t)\right|_{1, \infty} d t\right), \quad t \in\left[0, T_{1}\right] .
$$

To pass to the limit in this estimate, we proceed as follows. By Theorem 1 we may derive a bound for the $u^{n}$ in $H^{s}(\Omega)$, uniform in $n$, implying weak convergence of the sequence in $H^{s}(\Omega)$. Also, by a routine $L^{2}$ energy estimate for the $u^{n}$ from the Euler equations, we may establish the strong convergence of the sequence to $u$ in $L^{2}(\Omega)$. Then, by interpolation and the uniqueness of limits, the sequence converges strongly to $u$ in $H^{r}(\Omega)$ for any $r<s$, implying in particular by Sobolev's theorem, since $s \geqq 3$, that it converges strongly in $W^{1, \infty}(\Omega)$. Using these facts we may pass to the limit in the above estimate, obtaining

$$
|u(t)|_{s} \leqq\left|u_{0}\right|_{s} \exp \left(C \int_{0}^{T_{1}}|u(t)|_{1, \infty} d t\right)
$$

for $u(t) \in H^{s}(\Omega), t \in\left[0, T_{1}\right]$.

We now start with (18), and, following the steps leading from (14) to (17), with $T=T_{1}$, we obtain

$$
|u(t)|_{H^{s}(\Omega)} \leqq K, \quad t \in\left[0, T_{1}\right]
$$


By this estimate and Theorem 1 , we may extend $u(t)$ in $H^{s}(\Omega)$ to $t \in\left[T_{1}, T_{2}\right]$, where $T_{2}=2 T_{1}$ since $u(0)$ and $u\left(T_{1}\right)$ have the same bound $2 K$ in $H^{3}(\Omega)$. As before, we may approximate $u\left(T_{1}\right)$ smoothly, producing a sequence of solutions, derive (14) on $\left[T_{1}, T_{2}\right]$ for these smooth approximating solutions, pass to the limit, and use (18) with $t=T_{1}$, obtaining this time

$$
\begin{aligned}
|u(t)|_{s} & \leqq\left|u\left(T_{1}\right)\right|_{s} \exp \left(C \int_{T_{1}}^{T_{2}}|u(t)|_{1, \infty} d t\right) \\
& \leqq\left|u_{0}\right|_{s} \exp \left(C \int_{0}^{T_{2}}|u(t)|_{1, \infty} d t\right), \quad t \in\left[T_{1}, T_{2}\right] .
\end{aligned}
$$

Continuing as in the last paragraph, with $T=T_{2}$, and using (19), we obtain

$$
|u(t)|_{s} \leqq K, \quad t \in\left[0, T_{2}\right] .
$$

This process of initializing on $[0, \hat{T}]$ and extending may be continued likewise, and since the initial state will thus always be bounded in $H^{3}(\Omega)$ by $K$, we shall, after a finite number of steps, obtain that $u(x, \hat{T}) \in H^{s}(\Omega)$, the desired contradiction.

\section{The Elliptic Estimate}

The proof of (6) requires the application of results from the theory of linear elliptic systems of partial differential equations to a certain elliptic system related to the Euler equations. In Sect. 2.1 we shall introduce this system, verify its ellipticity, and list the necessary results, and in Sect. 2.2 we shall formally state and prove the estimate.

2.1. The Elliptic System. In order to derive (6), it would seem natural to consider the system

$$
\begin{array}{ll}
\nabla \times u=\omega & \text { in } \Omega \\
\nabla \cdot u=0 & \text { in } \Omega \\
u \cdot n=0 & \text { on } \partial \Omega,
\end{array}
$$

but instead we consider the following enlarged system, the function $\varphi$ being an additional scalar unknown:

$$
\begin{aligned}
\nabla \times u-\nabla \varphi=\zeta & \text { in } \Omega \\
\nabla \cdot u=\sigma & \text { in } \Omega \\
u \cdot n=\beta & \text { on } \partial \Omega \\
\varphi=\gamma & \text { on } \partial \Omega .
\end{aligned}
$$

This system, unlike (20), has the advantage of being elliptic. This will be verified in Lemma 3 below, but it is worth mentioning in passing that this system, unlike (20), corresponds to the exterior differential operator $d+\delta$, which is well known to be elliptic with the corresponding boundary conditions. 
For notational convenience we shall herein write (21) as follows, with $v=(u, \varphi)$, $\psi=(\zeta, \sigma)$, and $\chi=(\beta, \gamma)$ :

$$
\begin{array}{ll}
\mathscr{L} v=\psi & \text { in } \Omega \\
\mathscr{B} v=\chi & \text { on } \partial \Omega .
\end{array}
$$

Lemma 3. The operator $\mathscr{L}$ is uniformly elliptic in the sense of Agmon, Douglis, and Nirenberg, and $\mathscr{B}$ is complementing with respect to $\mathscr{L}$.

Proof. The symbols of $\mathscr{L}$ and $\mathscr{B}$ are, respectively,

$$
\begin{gathered}
\left(l_{i j}(x, \xi)\right)=\left(\begin{array}{cccc}
0 & -\xi_{3} & \xi_{2} & -\xi_{1} \\
\xi_{3} & 0 & -\xi_{1} & -\xi_{2} \\
-\xi_{2} & \xi_{1} & 0 & -\xi_{3} \\
\xi_{1} & \xi_{2} & \xi_{3} & 0
\end{array}\right), \\
\left(B_{h j}(x, \xi)\right)=\left(\begin{array}{cccc}
n_{1}(x) & n_{2}(x) & n_{3}(x) & 0 \\
0 & 0 & 0 & 1
\end{array}\right),
\end{gathered}
$$

for $\xi \in \mathbf{R}^{3}$.

To verify that $\mathscr{L}$ is uniformly elliptic, we verify the conditions of the definition on pp. 38-39 of [3]. Using the notation of that definition, we let $s_{i}=0$ for $i=1, \ldots, 4$, and $t_{j}=1$ for $j=1, \ldots, 4$. Then $s_{i} \leqq 0, t_{j} \geqq 0, \operatorname{deg} l_{i j}(x, \xi) \leqq s_{i}+$ $t_{j}=1$, for all $i$ and $j$, and since in this case $l_{i j}^{\prime}(x, \xi)=l_{i j}(x, \xi), \operatorname{det}\left(l_{i j}^{\prime}(\xi)\right)=|\xi|^{4}$, and $\mathscr{L}$ is thus uniformly elliptic.

To verify that $\mathscr{B}$ is complementing with respect to $\mathscr{L}$, we verify the conditions of the definition on pp. 42-43 of [3]. Using the notation of that definition, we let $r_{h}=-1$ for $h=1,2$, which implies that $\operatorname{deg} B_{h j}(x, \xi) \leqq r_{h}+t_{j}=0$, for all $h$ and $j$. Then noting that $\operatorname{det} l_{i j}^{\prime}(x, \zeta+\tau n)=(\tau-i|\zeta|)^{2}(\tau+i|\zeta|)^{2}, M^{+}(x, \zeta, \tau)=$ $(\tau-i|\zeta|)^{2}$, and $L^{j k}=-|\xi|^{2} l_{j k}^{\prime}$ since $l_{i j}^{\prime}$ is antisymmetric and has columns which are orthogonal and of norm $|\xi|$, we find that the rows of the matrix $\sum_{j=1}^{N} B_{h j}^{\prime}(x, \zeta+\tau n) L^{j k}(x, \zeta+\tau n)$ are linearly independent modulo $M^{+}$if and only if the following system of equations satisfied for all $\tau \in \mathbf{C}$ implies that $C_{1}=C_{2}=0$ :

$$
\begin{array}{r}
2 i|\zeta|(\tau-i|\zeta|)\left[C_{1}\left(-n_{2} \zeta_{3}+n_{3} \zeta_{2}\right)-C_{2}\left(\zeta_{1}+i|\zeta| n_{1}\right)\right]=0 \\
2 i|\zeta|(\tau-i|\zeta|)\left[C_{1}\left(n_{1} \zeta_{3}-n_{3} \zeta_{1}\right)-C_{2}\left(\zeta_{2}+i|\zeta| n_{2}\right)\right]=0 \\
2 i|\zeta|(\tau-i|\zeta|)\left[C_{1}\left(-n_{1} \zeta_{2}+n_{2} \zeta_{1}\right)-C_{2}\left(\zeta_{3}+i|\zeta| n_{3}\right)\right]=0 \\
-2 C_{1}|\zeta|^{2}(\tau-i|\zeta|)=0 .
\end{array}
$$

Since $\zeta$ and $n$ are linearly independent and $\zeta \neq 0$ by hypothesis, it is clear that this system holds identically in $\tau$ if and only if $C_{1}=C_{2}=0$. Therefore, $\mathscr{B}$ is complementing with respect to $\mathscr{L}$.

The system (21) is actually elliptic in a stronger sense than that of Agmon, Douglis, and Nirenberg, since $s_{i}=0$ and $r_{h}<0$ for all $i$ and $h$. An example of a system which is elliptic only in the more general sense is the Stokes system.

We now list some results that will be necessary in the next section. The following standard a-priori estimate for solutions of a certain class of first order linear elliptic systems is a specialization of Theorem 10.5 of [3]. 
Theorem 3. Let $\mathscr{D} \subseteq \mathbf{R}^{3}$ be a bounded domain of class $C^{1}$, and $u \in W^{1, p}(\mathscr{D})$ be a solution of

$$
\begin{array}{ll}
L u=f & \text { in } \mathscr{D} \\
B u=g & \text { on } \partial \mathscr{D},
\end{array}
$$

where $L$ is an $N \times N$ matrix partial differential operator uniformly elliptic in the sense of Agmon, Douglis, and Nirenberg with $s_{i}=0,1 \leqq i \leqq N, t_{j}=1,1 \leqq j \leqq N$, and coefficients in $C(\overline{\mathscr{D}}) ; B$ is an $m \times N$ matrix partial differential operator complementing with respect to $L$ with $r_{h}=-1,1 \leqq h \leqq m$, and coefficients in $C^{1}(\overline{\mathscr{D}})$; and $f \in L^{p}(\mathscr{D})$ and $g \in W^{1-1 / p, p}(\partial \mathscr{D}), 1<p<\infty$. Then there exists a constant $C$ not depending on $u$ such that

$$
|u|_{W^{1, p}(\mathscr{D})} \leqq C\left(|f|_{L^{p}(\mathscr{D})}+|g|_{W^{1-\frac{1}{p}, p(\partial \mathscr{D})}}+|u|_{L^{p}(\mathscr{D})}\right) .
$$

If the solution is unique in $W^{1, p}(\mathscr{D})$, then the last term on the right may be omitted, but with perhaps a different constant $C$.

The following uniqueness lemma will be necessary.

Lemma 4. If $\mathscr{D}$ is a bounded, simply connected domain with $\partial \mathscr{D} \in C^{2}$, and if $v \in W^{1, p}(\mathscr{D})$ is a solution of $(22)$ with $\Omega=\mathscr{D}$, then the solution is unique in $W^{1, p}(\mathscr{D})$.

Proof. We let $v_{0}=\left(u_{0}, \varphi_{0}\right)$ be a solution in $W^{1, p}(\mathscr{D})$ of (22) with homogeneous right-hand side, and show that $v_{0} \equiv 0$. Since $v_{0}$ satisfies the homogeneous system and is in $W^{1, p}(\mathscr{D})$, estimate (24) implies that it is of arbitrary smoothness, certainly in $C^{2}(\mathscr{D}) \cap C^{0}(\overline{\mathscr{D}})$. Applying the divergence operator to the first equation and utilizing the fourth equation we see that $\varphi_{0}$ is harmonic and vanishes on the boundary, implying that $\varphi_{0} \equiv 0$, and hence that $v_{0}=\left(u_{0}, 0\right)$. The homogeneous system then becomes

$$
\begin{aligned}
\nabla \times u_{0} & =0, \\
\nabla \cdot u_{0} & =0, \\
\left.u_{0} \cdot n\right|_{\partial} & =0 .
\end{aligned}
$$

Since $\mathscr{D}$ is simply connected, (25) implies that there exists a function $q \in C^{3}(\mathscr{D})$ such that $u_{0}=\nabla q$. Equations (26) and (27) then imply that $q$ satisfies a homogeneous Neumann problem, giving us that $q \equiv$ const., and hence that $u_{0} \equiv 0$. Therefore $v_{0} \equiv 0$.

The following theorem, supplying integral representations for solutions of first order linear elliptic systems of the type described in Theorem 3, along with pointwise estimates of the singular kernels, is a specialization of Theorem 1.1 of [11].

Theorem 4. Let $\mathscr{D} \subseteq \mathbf{R}^{3}$ be a bounded domain of class $C^{1}$, and $u$ be the unique solution in $C^{1+\alpha}(\mathscr{D})$ of $(23)$, but with $f \in C^{\alpha}(\mathscr{D})$ and $g \equiv 0,0<\alpha<1$. Then there exist a matrix function $\mathscr{G}_{\mathscr{D}}$ and a constant $C$, neither depending on $u$ or $f$, such that for all $x, y \in \mathscr{D}$, and every multiindex $\beta$,

$$
\begin{gathered}
u(x)=\int_{\mathscr{D}} \mathscr{G}_{\mathscr{D}}(x, y) f(y) d y, \text { and } \\
\left|D_{x}^{\beta} \mathscr{G}_{\mathscr{D}}(x, y)\right| \leqq C|x-y|^{-2-|\beta|} .
\end{gathered}
$$

2.2. Proof of Estimate. We now state and prove the main lemma of the paper. 
Proposition 1. If $\Omega \subseteq \mathbf{R}^{3}$ is a bounded, simply connected domain of class $C^{2+\beta}$, $0<\beta<1$, and if $v \in H^{3}(\Omega)$ is a solution of $(22)$ with $\psi \in H^{2}(\Omega)$ and $\chi \equiv 0$, then

$$
|v|_{W^{1, \infty}(\Omega)} \leqq C\left(1+\log +\frac{|\psi|_{H^{2}(\Omega)}}{|\psi|_{L^{\infty}(\Omega)}}\right)|\psi|_{L^{\infty}(\Omega)} .
$$

Corollary 1. If $u$ is a solution of the Euler equations as described in Theorem 2 and $\omega=\nabla \times u$, then for $t \in[0, \hat{T}]$,

$$
|u(\cdot, t)|_{W^{1, \infty}(\Omega)} \leqq C\left[\left(1+\log ^{+}|u(\cdot, t)|_{H^{s}(\Omega)}\right)|\omega(\cdot, t)|_{L^{\infty}(\Omega)}+1\right] .
$$

Proof of Corollary. Since $u$ satisfies the Euler equations and $\omega=\nabla \times u, v=(u, 0)$ clearly satisfies $(22)$ with $\psi=(\omega, 0)$ and $\chi=(0,0)$, and therefore (31) follows easily from (30).

Proof of Proposition. We obtain the estimate for $|v|_{1, \infty}$ by first estimating $\left|D_{k} v\right|_{\infty}$, $1 \leqq k \leqq 3$, from an integral representation for $v$, and later combining it with an easy estimate for $|v|_{\infty}$.

Proceeding with the former estimate, we obtain an integral representation for $v$ by appealing to Theorem 4 . Assuming for now that $v \in C^{3}(\Omega)$ and $\psi \in C^{2}(\Omega)$, we verify the hypotheses of that theorem. By assumption, $\partial \Omega$ and $v$ are sufficiently smooth, and the latter satisfies

$$
\begin{array}{ll}
\mathscr{L} v=\psi & \text { in } \Omega \\
\mathscr{B} v=0 & \text { on } \partial \Omega,
\end{array}
$$

which is a system of the required type by Lemma 3 and its proof, the smoothness of $\psi$, and the smoothness of the normal map for $\partial \Omega$ resulting from the smoothness of $\partial \Omega$ itself. Theorem 4 therefore guarantees the existence of a matrix function $\mathscr{G}_{\Omega}$ and a constant $C$, neither depending on $v$, such that for all $x, y \in \Omega$,

$$
\begin{gathered}
v(x)=\int_{\Omega} \mathscr{G}_{\Omega}(x, y) \psi(y) d y, \text { and } \\
\left|D_{x}^{\alpha} \mathscr{G}_{\Omega}(x, y)\right| \leqq C|x-y|^{-2-|\alpha|} .
\end{gathered}
$$

We estimate $\left|D_{k} v\right|_{\infty}$ directly from (33), separating the cases where $x$ is near or away from $\partial \Omega$. To that end, we let $\varepsilon$ be a small number, $0<\varepsilon \leqq 1$, depending only on $\Omega$, to be fixed later; and we proceed first with the interior estimate by choosing $x$ such that $\operatorname{dist}(x, \partial \Omega) \geqq 3 \varepsilon$.

Crucial to the special nature of the estimate (30) is the introduction of a small parameter $\delta, \delta \leqq \varepsilon<1$, which depends on the solution $v$, and which will be fixed later. Using $\delta$, we define

$$
\eta(y)= \begin{cases}1, & y \in B(x, \delta) \\ 0, & y \in \mathbf{R}^{3} \backslash B(x, 2 \delta),\end{cases}
$$

where $|\eta|_{\infty} \leqq 1$ and $\left|D_{k} \eta\right|_{\infty} \leqq C \delta^{-1}$, for all $k$. We then rewrite (33) in the form

$$
\begin{aligned}
v(x) & =\int_{\Omega} \mathscr{G}_{\Omega}(x, y) \eta(y) \psi(y) d y+\int_{\Omega} \mathscr{G}_{\Omega}(x, y)[1-\eta(y)] \psi(y) d y \\
& =v_{1}(x)+v_{2}(x),
\end{aligned}
$$

and estimate the terms separately. 
Using (34), we have for $k=1,2,3$,

$$
\begin{aligned}
\left|D_{k} v_{2}(x)\right| & =\left|\int_{\Omega} D_{x_{k}} \mathscr{G}_{\Omega}(x, y)[1-\eta(y)] \psi(y) d y\right| \\
& \leqq \sum_{i, j=1}^{4} \int_{\Omega \backslash(x, \delta)} C|x-y|^{-3}\left|\psi_{j}(y)\right| d y \\
& \leqq \bar{C}|\psi|_{L^{\infty}(\Omega)} \int_{\delta<|x-y|<R}|x-y|^{-3} d y \\
& \leqq\left(C_{1}-C_{2} \log \delta\right)|\psi|_{\infty},
\end{aligned}
$$

where $R=\max \{1, \operatorname{diam} \Omega\}$.

To estimate $\left|D_{k} v_{1}\right|_{\infty}$, we would like to apply the derivative to the first integral in (36) and integrate by parts, but this latter step is impossible by the asymmetry of $\mathscr{G}_{\Omega}$. Instead, we proceed in the following way. We note that by (36) $v_{1}$ satisfies

$$
\begin{aligned}
& \mathscr{L} v_{1}=\eta \psi \quad \text { in } \Omega \\
& \mathscr{B} v_{1}=0 \quad \text { on } \partial \Omega,
\end{aligned}
$$

and we localize this problem:

$$
\begin{aligned}
& \mathscr{L}\left(\zeta v_{1}\right)=\zeta \eta \psi+F \quad \text { in } \Omega \\
& \mathscr{B}\left(\zeta v_{1}\right)=0 \quad \text { on } \partial \Omega .
\end{aligned}
$$

Here $F=\left(\nabla \zeta \times u_{1}-\varphi_{1} \nabla \zeta, \nabla \zeta \cdot u_{1}\right), v_{1}=\left(u_{1}, \varphi_{1}\right)$, and $\zeta \in C_{0}^{\infty}\left(\mathbf{R}^{3}\right)$, with $|\zeta|_{\infty} \leqq 1$, $\zeta \equiv 1$ on $B(x, \varepsilon)$, and $\operatorname{supp} \zeta \subseteq B(x, 3 \varepsilon)$.

Utilizing $\mathscr{G}_{\Omega}$ once again, we may write for all $z \in \Omega$,

$$
\left(\zeta v_{1}\right)(z)=\int_{\Omega} \mathscr{G}_{\Omega}(z, y)[\zeta \eta \psi+F](y) d y .
$$

Since $\zeta \eta \psi+F \in C^{1+\beta}$, we may define

$$
r(z)=\int_{\Omega} \mathscr{G}_{\Omega}(z, y) D_{y_{k}}[\zeta \eta \psi+F](y) d y,
$$

where evidently $r \in C^{2+\beta}$, and satisfies

$$
\begin{aligned}
& \mathscr{L} r=D_{k}(\zeta \eta \psi+F) \text { in } \Omega \\
& \mathscr{B} r=0 \text { on } \partial \Omega .
\end{aligned}
$$

In turn, we define

$$
E=D_{k}\left(\zeta v_{1}\right)-r,
$$

and thus $E \in C^{1+\beta}$, and by (42) and (39) satisfies

$$
\begin{aligned}
\mathscr{L} E & =\mathscr{L}\left[D_{k}\left(\zeta v_{1}\right)-r\right] \\
& =\mathscr{L} D_{k}\left(\zeta v_{1}\right)-D_{k}(\zeta \eta \psi+F) \\
& =\left[\mathscr{L} D_{k}-D_{k} \mathscr{L}\right]\left(\zeta v_{1}\right)=0 \quad \text { in } \Omega \\
\mathscr{B} E & =\mathscr{B}\left[D_{k}\left(\zeta v_{1}\right)-r\right]=0 \quad \text { on } \partial \Omega,
\end{aligned}
$$


since $\mathscr{L}$ is constant coefficient and since $\zeta v_{1}$ vanishes in a neighborhood of $\partial \Omega$. By Lemma $4, E \equiv 0$, so that (43) becomes

$$
D_{k}\left(\zeta v_{1}\right)(z)=r(z)=\int_{\Omega} \mathscr{G}_{\Omega}(z, y) D_{y_{k}}[\zeta \eta \psi+F](y) d y .
$$

Proceeding with the estimate of $D_{k} v_{1}$, we simplify matters by separating the right-hand side of (45) into two terms, one containing the highest order terms and the terms depending on $\delta$, and the other containing only lower order terms independent of $\delta$, and we estimate each term separately:

$$
\begin{aligned}
D_{k}\left(\zeta v_{1}\right)(z)= & \int_{\Omega} \mathscr{G}_{\Omega}(z, y)\left[\zeta \psi D_{k} \eta+\eta \zeta D_{k} \psi\right](y) d y \\
& +\int_{\Omega} \mathscr{G}_{\Omega}(z, y)\left[\eta \psi D_{k} \zeta+D_{k} F\right](y) d y \\
= & w_{1}(z)+w_{2}(z)
\end{aligned}
$$

To estimate $w_{2}$, we note from (46) the boundary value problem it satisfies, and use the Sobolev Embedding Theorem, (24), (35), (38), and the definitions below (39), to obtain for $3<p<\infty$,

$$
\begin{aligned}
\left|w_{2}\right|_{L^{\infty}(\Omega)} & \leqq C\left|w_{2}\right|_{W^{1, p}(\Omega)} \\
& \leqq C\left|\eta \psi D_{k} \zeta+D_{k} F\right|_{L^{p}(\Omega)} \\
& \leqq C\left(|\psi|_{L^{p}(\Omega)}+\left|v_{1}\right|_{W^{1, p}(\Omega)}\right) \\
& \leqq C\left(|\psi|_{L^{p}(\Omega)}+|\eta \psi|_{L^{p}(\Omega)}\right) \leqq C|\psi|_{L^{\infty}(\Omega)},
\end{aligned}
$$

where $C$ is independent of $\delta$. To estimate $w_{1}$, we utilize its integral representation in (46) directly, evaluated at $z=x$, using (34), (35), the fact that supp $\zeta \subseteq B(x, 3 \varepsilon)$, Hölder's inequality, and Sobolev's theorem:

$$
\begin{aligned}
\left|w_{1}(x)\right| \leqq & \sum_{j=1}^{4} \int_{|x-y|<2 \delta} C|x-y|^{-2}\left|\left(D_{k} \psi_{j}\right)(y)\right| d y \\
& +\sum_{j=1}^{4} \int_{\delta<|x-y|<2 \delta} C \delta^{-1}|x-y|^{-2}\left|\psi_{j}(y)\right| d y \\
\leqq & C\left|D_{k} \psi\right|_{L^{6}(\Omega)}\left(\int_{r<2 \delta} r^{\frac{-12}{5}} r^{2} d r\right)^{\frac{5}{6}} \\
& +C \delta^{-1}|\psi|_{L^{\infty}(\Omega)} \int_{\delta<r<2 \delta} r^{-2} r^{2} d r \\
\leqq & C\left(\delta^{\frac{1}{2}}\left|D_{k} \psi\right|_{L^{6}(\Omega)}+|\psi|_{L^{\infty}(\Omega)}\right) \\
\leqq & C\left(\delta^{\frac{1}{2}}|\psi|_{H^{2}(\Omega)}+|\psi|_{L^{\infty}(\Omega)}\right) .
\end{aligned}
$$

Hölder's inequality is applied in line three, and Sobolev's theorem in line six. Combining (47) and (48) into (46), and using the fact that $\zeta \equiv 1$ on $B(x, \varepsilon)$, we obtain for $k=1,2,3$,

$$
\left|D_{k} v_{1}(x)\right|=\left|D_{k}\left(\zeta v_{1}\right)(x)\right| \leqq C\left(\delta^{\frac{1}{2}}|\psi|_{H^{2}(\Omega)}+|\psi|_{L^{\infty}(\Omega)}\right) .
$$


And combining (49) and (37) into (36), we obtain the interior estimate, for $k=1,2$, $3, \delta \leqq \varepsilon$, and $x \in \Omega$ such that $\operatorname{dist}(x, \partial \Omega) \geqq 3 \varepsilon$ :

$$
\left|D_{k} v(x)\right| \leqq\left(C_{1}-C_{2} \log \delta\right)|\psi|_{L^{\infty}(\Omega)}+C \delta^{\frac{1}{2}}|\psi|_{H^{2}(\Omega)}
$$

We turn now to the boundary estimate, choosing $x$ such that $\operatorname{dist}(x, \partial \Omega)<3 \varepsilon$. As in the interior case, we use (36) and estimate the two terms separately. $D_{k} v_{2}$ is estimated almost exactly as in (37), yielding for $k=1,2,3$,

$$
\left|D_{k} v_{2}(x)\right| \leqq\left(C_{1}-C_{2} \log \delta\right)|\psi|_{L^{\infty}(\Omega)} .
$$

To estimate $\left|D_{k} v_{1}\right|_{\infty}$, we proceed similarly to the interior case, but with two important modifications: we apply a change of coordinates to the local problem, flattening the boundary, and we estimate only tangential derivatives directly, the normal derivative being estimated indirectly from the equations.

Before proceeding with the estimate, we must define the neighborhood of $x$ in which the localization of (38) will take place, so that it is contained in a coordinate patch of $\partial \Omega$ and so that its boundary is smooth. To this end, we first fix $\varepsilon$ as follows, so that $B^{*}(x, 3 \varepsilon)=B(x, 3 \varepsilon) \cap \Omega$ is contained in one of the coordinate patches of $\partial \Omega$. Since $\partial \Omega$ is $C^{2+\alpha}$, for every $y \in \partial \Omega$ there exists an open set $U_{y}$ containing $y$, and a $C^{2+\alpha}$ diffeomorphism $\Phi_{y}: U_{y} \rightarrow B(0,1) \subseteq \mathbf{R}^{3}$ that maps $\Omega \cap U_{y}$ into the halfspace $\mathbf{R}_{+}^{3}=\left\{\left(x_{1}, x_{2}, x_{3}\right) \in \mathbf{R}^{3}: x_{3}>0\right\}$, and $\partial \Omega \cap U_{y}$ into $x_{3}=0$. For technical reasons, we consider the neigborhoods $\widetilde{U}_{y}=\Phi_{y}^{-1}\left(B\left(0, \frac{1}{2}\right)\right)$. For each $\tilde{U}_{y}$, choose a ball $B\left(y, r_{y}\right)$ contained in $\tilde{U}_{y}$, and cover $\partial \Omega$ with $\left\{B\left(y, r_{y} / 4\right)\right\}_{y \in \partial \Omega}$. Now choose a finite subcover $\left\{B\left(y_{l}, r_{l} / 4\right)\right\}_{l=1}^{M}$, let $d=\min \left\{r_{l} / 4\right\}$, and let $\varepsilon=\min \{1, d / 9\}$. Then it is clear that for any $x$ such that $\operatorname{dist}(x, \partial \Omega)<3 \varepsilon, B^{*}(x, 3 \varepsilon) \subseteq \tilde{U}_{l}$ for some $l$, since if $\tilde{y}$ is a point on $\partial \Omega$ closest to $x$, then $\operatorname{dist}(\tilde{y}, x)<3 \varepsilon \leqq d / 3$, and $B^{*}(x, 3 \varepsilon) \subseteq B(x, 3 \varepsilon) \subseteq$ $B(x, d / 3) \subseteq B(\tilde{y}, d) \subseteq B\left(y_{l}, r_{l} / 2\right) \subseteq \tilde{U}_{l}$, for some $l$.

Neither $B^{*}(x, 3 \varepsilon)$ nor its image $\Phi_{l}\left(B^{*}(x, 3 \varepsilon)\right)$ has smooth boundary, but since $\Phi_{l}\left(B^{*}(x, 3 \varepsilon)\right) \subseteq B\left(0, \frac{1}{2}\right)$, it is clear that we may construct a neighborhood $\tilde{N} \subseteq \mathbf{R}_{+}^{3}$ which is simply connected and has $C^{2+\beta}$ boundary, such that $\Phi_{l}\left(B^{*}(x, 3 \varepsilon)\right) \subseteq$ $B\left(0, \frac{1}{2}\right) \subseteq \tilde{N} \subseteq B(0,1)$. Its inverse image $N=\Phi_{l}^{-1}(\widetilde{N})$ is clearly also simply connected and has $C^{2+\beta}$ boundary, with $B^{*}(x, 3 \varepsilon) \subseteq N \subseteq U_{l} \cap \Omega$.

We formulate the following local version of $(38)$ in $N$ :

$$
\begin{aligned}
& \mathscr{L}\left(\zeta v_{1}\right)=\zeta \eta \psi+F \quad \text { in } N \\
& \mathscr{B}\left(\zeta v_{1}\right)=0 \text { on } \partial N .
\end{aligned}
$$

Here $F$ and $\zeta$ are defined as in (39). The boundary operator $\mathscr{B}$ is well-defined and has $C^{1+\beta}$ coefficients by the smoothness of $\partial \Omega$, and the boundary condition is satisfied since $\zeta \equiv 0$ outside $B(x, 3 \varepsilon)$ and since $\mathscr{B} v_{1} \equiv 0$ on $\partial \Omega$. Applying $\Phi_{l}$ to (52), we obtain

$$
\begin{aligned}
& \tilde{\mathscr{L}}\left(\tilde{\zeta} \tilde{v}_{1}\right)=\tilde{\zeta} \tilde{\eta} \tilde{\psi}+\tilde{F} \text { in } \tilde{N}, \\
& \tilde{\mathscr{B}}\left(\tilde{\zeta}_{1}\right)=0 \text { on } \partial \tilde{N},
\end{aligned}
$$

where $\tilde{f}=f \circ \Phi_{l}$ for each function $f$ above, and $\tilde{\mathscr{L}}$ and $\tilde{\mathscr{B}}$ are the operators obtained from $\mathscr{L}$ and $\mathscr{B}$ through $\Phi_{l}$.

As in the interior case, we obtain an integral representation for $\tilde{\zeta} \tilde{v}_{1}$ by appealing to Theorem 4 , the hypotheses of which we now verify. By construction, $\tilde{N}$ is 
bounded and has the necessary smoothness, and the system (53) is of the required type since the uniform ellipticity and complementing condition, as well as the smoothness of the coefficients and the right-hand side, are preserved under the smooth coordinate map $\Phi_{l}$ (see [3], for instance). In addition, $\widetilde{\zeta} \tilde{v}_{1}$ is the unique solution of (53) since by Lemma $4, \zeta v_{1}$ is the unique solution of (52). Theorem 4 therefore guarantees the existence of a matrix function $\mathscr{G}_{\tilde{N}}$ and a constant $\tilde{C}$, neither depending on $\widetilde{\zeta} \tilde{v}_{1}$, such that for all $\tilde{y}, \tilde{z} \in \tilde{N}$,

$$
\begin{gathered}
\left(\tilde{\zeta} \tilde{v}_{1}\right)(\tilde{z})=\int_{\tilde{N}} \mathscr{G}_{\tilde{N}}(\tilde{z}, \tilde{y})[\tilde{\zeta} \tilde{\eta} \tilde{\psi}+\tilde{F}](\tilde{y}) d \tilde{y}, \text { and } \\
\left|\tilde{D}_{\tilde{z}}^{\alpha} \mathscr{G}_{\tilde{N}}(\tilde{z}, \tilde{y})\right| \leqq \tilde{C}|\tilde{z}-\tilde{y}|^{-2-|\alpha|}
\end{gathered}
$$

We estimate only the tangential derivatives $\tilde{D}_{k}, k=1,2$, of $\tilde{\zeta}_{1}$, directly from (54). To this end we must, as in the interior case, apply the following procedure, in lieu of an integration by parts. Since $\tilde{\zeta} \tilde{\eta} \tilde{\psi}+\widetilde{F} \in C^{1+\beta}$, we define

$$
\tilde{r}(\tilde{z})=\int_{\tilde{N}} \mathscr{G}_{\tilde{N}}(\tilde{z}, \tilde{y}) D_{\tilde{y}_{k}}[\tilde{\zeta} \tilde{\eta} \tilde{\psi}+\tilde{F}](\tilde{y}) d \tilde{y},
$$

where evidently $\tilde{r} \in C^{1+\beta}$ and satisfies

$$
\begin{aligned}
& \tilde{\mathscr{L}} \tilde{r}=\tilde{D}_{k}(\tilde{\zeta} \tilde{\eta} \tilde{\psi}+\tilde{F}) \text { in } \tilde{N}, \\
& \tilde{\mathscr{B}} \tilde{r}=0 \quad \text { on } \partial \tilde{N} .
\end{aligned}
$$

In turn, we define

$$
\tilde{E}=\tilde{D}_{k}\left(\tilde{\zeta} \tilde{v}_{1}\right)-\tilde{r},
$$

and therefore $\tilde{E} \in C^{1+\beta}$, and by (57) and (53) satisfies

$$
\begin{aligned}
\tilde{\mathscr{L}} \tilde{E} & =\tilde{\mathscr{L}}\left[\tilde{D}_{k}\left(\tilde{\zeta} \tilde{v}_{1}\right)-\tilde{r}\right] \\
& =\tilde{\mathscr{L}} \tilde{D}_{k}\left(\tilde{\zeta} \tilde{v}_{1}\right)-\tilde{D_{k}}(\tilde{\zeta} \tilde{\eta} \tilde{\psi}+\tilde{F}) \\
& =\left[\tilde{\mathscr{L}} \tilde{D}_{k}-\tilde{D_{k}} \tilde{\mathscr{L}}\right]\left(\tilde{\zeta} \tilde{\zeta}_{1}\right) \quad \text { in } \tilde{N}, \\
\tilde{\mathscr{B}} \tilde{E} & =\tilde{\mathscr{B}}\left[\tilde{D}_{k}\left(\tilde{\zeta} \tilde{v}_{1}\right)-\tilde{r}\right] \\
& =\tilde{\mathscr{B}} \tilde{D}_{k}\left(\tilde{\zeta} \tilde{v}_{1}\right) \\
& =\left[\tilde{\mathscr{B}} \tilde{D}_{k}-\tilde{D_{k}} \tilde{\mathscr{B}}\right]\left(\tilde{\zeta} \tilde{v}_{1}\right) \text { on } \partial \tilde{N} .
\end{aligned}
$$

The last equality holds since $\tilde{D_{k}}$ is tangential to the flat portion of $\partial \tilde{N}$, where $\tilde{\mathscr{B}}\left(\tilde{\zeta} \tilde{v}_{1}\right)$ vanishes, and also since $\tilde{\zeta} \tilde{v}_{1}$ vanishes on a neighborhood of the irregular portion of $\partial N$. It should be mentioned that although $\widetilde{E}$ is in general not identically equal to zero, as was $E$ in the interior case, it does turn out to be of lower order than $\tilde{r}$, and hence is easily estimated. Therefore (58) remains

$$
\tilde{D}_{k}\left(\tilde{\zeta} \tilde{\zeta}_{1}\right)(\tilde{z})=\int_{\tilde{N}} \mathscr{G}_{\tilde{N}}(\tilde{z}, \tilde{y}) D_{\tilde{y}_{k}}[\tilde{\zeta} \tilde{\eta} \tilde{\psi}+\tilde{F}](\tilde{y}) d \tilde{y}+\tilde{E}(\tilde{z}) .
$$


Proceeding with the estimate of the tangential derivatives, we rewrite (60) as in (46), and estimate the terms separately:

$$
\begin{aligned}
\tilde{D}_{k}\left(\tilde{\zeta} \tilde{v}_{1}\right)(\tilde{z})= & \int_{\tilde{N}} \mathscr{G}_{\tilde{N}}(\tilde{z}, \tilde{y})\left[\tilde{\zeta} \tilde{\psi} \tilde{D_{k}} \tilde{\eta}+\tilde{\eta} \tilde{\zeta} \tilde{D_{k}} \tilde{\psi}\right](\tilde{y}) d \tilde{y} \\
& +\int_{\tilde{N}} \mathscr{G}_{\tilde{N}}(\tilde{z}, \tilde{y})\left[\tilde{\eta} \tilde{\psi} \tilde{D_{k}} \tilde{\zeta}+\tilde{D_{k}} \tilde{F}\right](\tilde{y}) d \tilde{y}+\tilde{E}(\tilde{z}) \\
= & \tilde{w}_{1}(\tilde{z})+\tilde{w}_{2}(\tilde{z})+\tilde{E}(\tilde{z}) .
\end{aligned}
$$

To estimate $\tilde{w}_{2}$, we proceed as in (47), so that we have for $3<p<\infty$,

$$
\left|\tilde{w}_{2}\right|_{L^{\infty}(\tilde{N})} \leqq C|\psi|_{L^{\infty}(\Omega)} .
$$

To estimate $\tilde{E}$, we use Sobolev's Theorem, (24), (59), (38), the Trace theorem, and the fact that the commutators are of one lower order than the products of the operators themselves, to obtain

$$
\begin{aligned}
|\tilde{E}|_{L^{\infty}(\tilde{N})} & \leqq C|\tilde{E}|_{W^{1, p}(\tilde{N})} \\
& \leqq C\left(\left|\left[\tilde{\mathscr{L}} \tilde{D}_{k}-\tilde{D}_{k} \tilde{\mathscr{L}}\right]\left(\tilde{\zeta} \tilde{v}_{1}\right)\right|_{L^{p}(\tilde{N})}+\left|\left[\tilde{\mathscr{B}} \tilde{D}_{k}-\tilde{D}_{k} \tilde{\mathscr{B}}\right]\left(\tilde{\zeta} \tilde{v}_{1}\right)\right|_{W^{1-\frac{1}{p}, p}(\partial \tilde{N})}\right) \\
& \leqq C\left|v_{1}\right|_{W^{1, p}(\Omega)} \\
& \leqq C|\eta \psi|_{L^{p}(\Omega)} \\
& \leqq C|\psi|_{L^{\infty}(\Omega)} .
\end{aligned}
$$

The constants appearing in (62) and (63) are independent of $\delta$ and can be made independent of $x$ because of the finiteness of the cover of $\partial \Omega$ and the construction of $\tilde{N}$. To estimate $\tilde{w}_{1}$, we utilize its integral representation in (61) directly, as in (48), evaluated at $\tilde{z}=\tilde{x}$ :

$$
\begin{aligned}
& \left|\tilde{w}_{1}(\tilde{x})\right| \leqq \sum_{i, j=1}^{4} \int_{\tilde{N}}\left|\tilde{G}_{i j}(\tilde{x}, \tilde{y})\right|\left|\left(\tilde{\eta} \tilde{\zeta} \tilde{D}_{k} \tilde{\psi}_{j}\right)(\tilde{y})\right| d \tilde{y} \\
& +\sum_{i, j=1}^{4} \int_{\tilde{N}}\left|\tilde{G}_{i j}(\tilde{x}, \tilde{y})\right|\left|\left(\tilde{\zeta} \tilde{\psi}_{j} \tilde{D}_{k} \tilde{\eta}\right)(\tilde{y})\right| d \tilde{y} \\
& \leqq \sum_{j=1}^{4} \int_{B^{*}(x, 3 \varepsilon)} C|x-y|^{-2}\left|\left(\eta \mathscr{D} \psi_{j}\right)(y)\right| d y \\
& +\sum_{j=1}^{4} \int_{B^{*}(x, 3 \varepsilon)} C|x-y|^{-2}\left|\left(\psi_{j} \mathscr{D} \eta\right)(y)\right| d y \\
& \leqq C|\mathscr{D} \psi|_{L^{6}(B(x, 2 \delta) \cap \Omega)}\left(\int_{r<2 \delta} r^{-12 / 5} r^{2} d r\right)^{\frac{5}{6}} \\
& +C \delta^{-1}|\psi|_{L^{\infty}(\Omega)} \int_{\delta<r<2 \delta} r^{-2} r^{2} d r \\
& \leqq C\left(\delta^{\frac{1}{2}}|\mathscr{D} \psi|_{L^{6}\left(\Phi^{-1}(\tilde{N})\right)}+|\psi|_{L^{\infty}(\Omega)}\right) \\
& \leqq C\left(\delta^{\frac{1}{2}}|\psi|_{H^{2}(\Omega)}+|\psi|_{L^{\infty}(\Omega)}\right) \text {. }
\end{aligned}
$$


In the above, $\mathscr{D}$ is the first order linear operator with coefficients from the Jacobian of $\Phi_{l}$, obtained through the chain rule. The constant $C$ can again be made independent of $x$.

Combining (62), (63), and (64) into (61), we obtain for $k=1,2$,

$$
\left|\tilde{D}_{k} \tilde{v}_{1}(\tilde{x})\right|=\left|\tilde{D}_{k}\left(\tilde{\zeta} \tilde{v}_{1}\right)(\tilde{x})\right| \leqq C\left(\delta^{\frac{1}{2}}|\psi|_{H^{2}(\Omega)}+|\psi|_{L^{\infty}(\Omega)}\right) .
$$

The remaining derivative ${\tilde{D_{3}}}_{3} \tilde{v}_{1}$ is estimated by solving for it in the system (21) in terms of previously estimated quantities. We first rewrite the interior equations of (21), letting $v_{1}=\left(u^{1}, \varphi^{1}\right)$ and $v_{2}=\left(u^{2}, \varphi^{2}\right)$ :

$$
\begin{aligned}
u_{2,3}^{1}-u_{3,2}^{1}-\varphi_{1}^{1} & =\omega_{1}-\left(\nabla \times u^{2}\right)_{1}+\varphi_{1}^{2}, \\
u_{3,1}^{1}-u_{1,3}^{1}-\varphi_{2}^{1} & =\omega_{2}-\left(\nabla \times u^{2}\right)_{2}+\varphi_{2}^{2}, \\
u_{1,2}^{1}-u_{2,1}^{1}-\varphi_{3}^{1} & =\omega_{3}-\left(\nabla \times u^{2}\right)_{3}+\varphi_{3}^{2}, \\
u_{1,1}^{1}+u_{2,2}^{1}+u_{3,3}^{1} & =-\nabla \cdot u^{2} .
\end{aligned}
$$

Considering the system in $U_{l}$, we apply $\Phi_{l}$ to the left-hand side, producing Jacobian terms $\tilde{x}_{i, j}$, and we solve for the terms containing the components of $\tilde{D}_{3} \tilde{v}_{1}$ :

$$
\begin{aligned}
\tilde{u}_{2,3}^{1} \tilde{x}_{3,3}-\tilde{u}_{3,3}^{1} \tilde{x}_{3,2}-\tilde{\varphi}_{3}^{1} \tilde{x}_{3,1} & =F, \\
\tilde{u}_{3,3}^{1} \tilde{x}_{3,1}-\tilde{u}_{1,3}^{1} \tilde{x}_{3,3}-\tilde{\varphi}_{3}^{1} \tilde{x}_{3,2} & =G, \\
\tilde{u}_{1,3}^{1} \tilde{x}_{3,2}-\tilde{u}_{2,3}^{1} \tilde{x}_{3,1}-\tilde{\varphi}_{3}^{1} \tilde{x}_{3,3} & =H, \\
\tilde{u}_{1,3}^{1} \tilde{x}_{3,1}+\tilde{u}_{2,3}^{1} \tilde{x}_{3,2}+\tilde{u}_{3,3}^{1} \tilde{x}_{3,3} & =K .
\end{aligned}
$$

The functions $F, G, H$, and $K$ evidently consist of previously estimated quantities such as $\omega$, derivatives of $v_{2}$, tangential derivatives of $\tilde{v}_{1}$, and Jacobian terms which are bounded independent of $l$. The determinant of the coefficient matrix for this system is $\left(\tilde{x}_{3,1}^{2}+\tilde{x}_{3,2}^{2}+\tilde{x}_{3,3}^{2}\right)^{2}$, which, because of the nonsingularity of the Jacobian, never vanishes. Therefore $\tilde{D}_{3} \tilde{v}_{1}=\left(\tilde{u}_{1,3}^{1}, \tilde{u}_{2,3}^{1}, \tilde{u}_{3,3}^{1}, \tilde{\varphi}_{3}^{1}\right)$ may be solved for in (67) and estimated in terms of previously estimated quantities, yielding

$$
\left|{\tilde{D_{3}}}_{3} \tilde{v}_{1}(\tilde{x})\right| \leqq C \delta^{\frac{1}{2}}|\psi|_{H^{2}(\Omega)}+\left(C_{1}-C_{2} \log \delta\right)|\psi|_{L^{\infty}(\Omega)} .
$$

This, combined with (65), yields the estimate for $D_{k} v_{1}, k=1,2,3$ :

$$
\left|D_{k} v_{1}(x)\right|=\left|\sum_{j=1}^{3}\left(\tilde{D}_{j} v_{1}\right) x_{j, k}\right| \leqq\left(C_{1}-C_{2} \log \delta\right)|\psi|_{L^{\infty}(\Omega)}+C \delta^{\frac{1}{2}}|\psi|_{H^{2}(\Omega)} .
$$

And, combining (51) and (69), we obtain the boundary estimate, for $k=1,2,3$, $\delta \leqq \varepsilon$, and $x \in \Omega$ such that $\operatorname{dist}(x, \partial \Omega)<3 \varepsilon$ :

$$
\left|D_{k} v(x)\right| \leqq\left(C_{1}-C_{2} \log \delta\right)|\psi|_{L^{\infty}(\Omega)}+C \delta^{\frac{1}{2}}|\psi|_{H^{2}(\Omega)} .
$$

The interior estimate (50) and the boundary estimate (70) combine to yield the global estimate for $\delta \leqq \varepsilon$ :

$$
\left|D_{k} v\right|_{L^{\infty}(\Omega)} \leqq\left(C_{1}-C_{2} \log \delta\right)|\psi|_{L^{\infty}(\Omega)}+C \delta^{\frac{1}{2}}|\psi|_{H^{2}(\Omega)} .
$$


The proof of the proposition is completed by setting $\delta=\min \left\{\left(\frac{|\psi|_{L^{\infty}(\Omega)}}{|\psi|_{H^{2}(\Omega)}}\right)^{2}, \varepsilon\right\}$, which in either case leads to

$$
\left|D_{k} v\right|_{L^{\infty}(\Omega)} \leqq C\left(1+\log ^{+} \frac{|\psi|_{H^{2}(\Omega)}}{|\psi|_{L^{\infty}(\Omega)}}\right)|\psi|_{L^{\infty}(\Omega)}
$$

Using Sobolev's theorem and (24) applied to (32), we have for $3<p<\infty$,

$$
|v|_{L^{\infty}(\Omega)} \leqq C|v|_{W^{1, p}(\Omega)} \leqq C|\psi|_{L^{p}(\Omega)} \leqq C|\psi|_{L^{\infty}(\Omega)},
$$

which combined with (72) finally gives us

$$
|v|_{W^{1, \infty}(\Omega)} \leqq C\left(1+\log +\frac{|\psi|_{H^{2}(\Omega)}}{|\psi|_{L^{\infty}(\Omega)}}\right)|\psi|_{L^{\infty}(\Omega)} .
$$

This estimate has been derived for $\psi \in C^{2}(\Omega)$ and $v \in C^{3}(\Omega)$, but a routine density argument establishes it for $\psi \in H^{2}(\Omega)$ and $v \in H^{3}(\Omega)$.

\section{References}

1. Adams, R.: Sobolev Spaces. New York: Academic Press 1975

2. Agmon, S., Douglis, A., Nirenberg, L.: Estimates near the boundary for solutions of elliptic partial differential equations satisfying general boundary conditions I. Commun. Pure Appl. Math. 17, 623-727 (1959)

3. Agmon, S., Douglis, A., Nirenberg, L.: Estimates over the boundary for solutions of elliptic partial differential equations satisfying general boundary conditions II. Commun. Pure Appl. Math. 17, 35-92 (1964)

4. Beale, J.T., Kato, T., Majda, A.: Remarks on the breakdown of Smooth Solutions for the 3-D Euler Equations. Commun. Math. Phys. 94, 61-66 (1984)

5. Ferrari, A.: On the Blow-up of Solutions of the 3-D Euler Equations in a Bounded Domain. Ph.D. thesis, Duke University, 1992

6. Kato, T., Lai, C.Y.: Nonlinear Evolution Equations and the Euler Flow. J. Funct. Anal. 56, $15-28(1984)$

7. Kato, T., Ponce, G.: Well-posedness of the Euler and Navier-Stokes equations in the Lebesgue Spaces $L_{s}^{p}\left(\mathbf{R}^{2}\right)$. Revista Matematicas Iberoamericana 2, 73-88 (1986)

8. Klainerman, S., Majda, A.: Singular limits of quasilinear hyperbolic systems with large parameters and the incompressible limit of compressible fluids. Commun. Pure Appl. Math. 34, 481-524 (1981)

9. Majda, A.: Compressible Fluid Flow and Systems of Conservation Laws in Several Space Variables. In: Applied Math. Sciences Series, Vol. 53, Berlin, Heidelberg, New York: Springer 1983

10. Majda, A.: Vorticity and the Mathematical Theory of Incompressible Fluid Flow. Lecture Notes, Princeton University, 1986-1987

11. Solonnikov, V.A.: On Green's Matrices for Elliptic Boundary Value Problems I. Trudy Mat. Inst. Steklov 110, 123-170 (1970)

12. Solonnikov, V.A.: On Green's Matrices for Elliptic Boundary Value Problems II. Trudy Mat. Inst. Steklov 116, 187-226 (1971)

13. Taylor, M.: Pseudodifferential Operators and Nonlinear PDE. Boston, MA: Birkhäuser 1991

14. Temam, R.: On the Euler Equations of Incompressible Perfect Fluids. J. Funct. Anal. 20, 32-43 (1975)

15. Wahl, W. von: Vorlesung über das Außenraumproblem für die instationären Gleichungen von Navier-Stokes. Rudolph-Lipschitz-Vorlesung, Sonderforschungsbereich 256. Nichtlineare Partielle Differentialgleichungen, Bonn, 1989 
16. Wahl, W. von: Estimating $\nabla u$ by $\nabla \cdot u$ and $\nabla \times u$. To appear in Math. Methods in the Appl. Sciences

17. Yanagisawa, T.: A Continuation Principle for the Euler Equations for Incompressible Fluids in a Bounded Domain. Preprint

18. Zajaczkowski, M.: Remarks on the Breakdown of Smooth Solutions for the 3-D Euler Equations in a Bounded Domain. Bull. Polish Acad. of Sciences Mathematics 37, 169-181 (1989)

Communicated by J.L. Lebowitz 